Por último, otra vez el inventario de Odescalchi nos proporciona la identificación del siguiente relieve que decoraba el pedestal de la llamada "Venus del baño» (Museo del Prado, $\mathrm{n} .{ }^{\circ}$ E-33) «....un bajorrelieve moderno, de 1,10 palmos de alto, representando un sátiro en el acto de abrazar a una mujen ${ }^{14}$, sin lugar a dudas el de la Granja (Fig. 8), «Pan y Sirinx», muy cerca del estilo de Monnot, aunque en otro inventario del mismo Odescalchi se cita como antiguo «de Pan que abraza a Sirinx, y que ésta se convierte en caña con su padre en el pantano en forma de río" ${ }^{15}$.

Los relieves localizados deberían ser restituidos al lugar que ocuparon durante tanto tiempo, los pedestales de las célebres esculturas que se conservan en el Museo del Prado, ya que si permanecieron en el palacio de la Granja fue porque no se valoraron lo suficiente en el momento del traslado.

Rosario Coppel Areizaga Dra. por la Universidad Complutense

\title{
APORTACIONES AL ESTUDIO DE LA MINIATURA GÓTICA ITALIANA: TRES FRAGMENTOS DE LA BIBLIOTECA NACIONAL
}

Dentro de la gran colección de dibujos de la Biblioteca Nacional se conserva un conjunto de seis miniaturas recortadas que abarca desde comienzos del siglo xIV a mediados del siglo XvI. Las más notables $(\mathrm{AB} / 1922, \mathrm{AB} / 1923$ y $\mathrm{AB} / 1925)$, son de origen italiano, mientras que las otras tres son españolas $\left(\mathrm{AB} / 1924, \mathrm{AB} / 1926\right.$ y AB/1927) ${ }^{1}$. Todas tienen aún restos de cola en el verso que indican que debieron estar pegadas a otro soporte y posiblemente enmarcadas. Salvo en el caso de la inicial AB/1926, que entró con la colección de Manuel Castellano, ignoramos su procedencia.

El coleccionismo de hojas sueltas iluminadas existió, especialmente en Flandes, desde el siglo xv, pero la mutilación de manuscritos con fines comerciales no nació hasta comienzos del siglo XVIII ${ }^{2}$. Al final de dicha centuria las invasiones napoleónicas de Italia y las subsiguientes secularizaciones de conventos y monasterios sacaron a la luz numerosos códices, especialmente libros corales, cuyo tamaño y escasa decoración los hacía inadecuados para el comercio. Se crearon entonces importantes colecciones de miniaturas recortadas entre las que destaca especialmente la del abate Luigi Celotti.

Esta forma de vandalismo, que privaba a las miniaturas de su significación histórica e incluso estética, continuó a lo largo de los siglos xix y xx, creándose numerosas colecciones. También se formaron en España, aunque con posterioridad al resto de Europa, y coleccionistas como Ma-

\footnotetext{
14 Documenti inediti IV, pp. 336-37.

15 Walker, op. cit., n. ${ }^{\circ} 62$.

1 Preparamos un estudio sobre estas tres miniaturas. Existe otro fragmento ya publicado. Se trata de un folio de un libro de horas flamenco con la figura de San Matías, que fue incluido por Ángel M. de Barcia en su Catálogo de la colección de dibujos originales de la Biblioteca Nacional, Madrid, 1906, con el número 8669. F. Winkler, en Die Flämische Buchmalerei des XV. und XVI. Jahrhunderts, Amsterdam, 1978, p. 129, lo atribuyó a Antoine Papin.

2 Voelkle, William M. Voelkle y Wieck, Roger S., The Bernard Breslauer Collection of manuscript illuminations, Nueva York, 1992, p. 13; Alexander, J. J. G., Wallace Collection. Catalogue of Illuminated Manuscript Cuttings, Londres, 1980 , p. 9.
} 
nuel Rico y Sinobas, José Lázaro Galdiano o Valentín de Carderera contaron entre sus fondos con este tipo de obras. Al igual que sus colegas europeos adquirieron sobre todo iniciales de cantorales italianos y hojas de libros de horas flamencos y franceses.

Los tres fragmentos que vamos a presentar corresponden a libros de coro, soporte característico de buena parte de la miniatura gótica italiana. Solían ser de gran tamaño y pueden dividirse en dos grupos: graduales, que contienen las partes corales de la misa y cuyo nombre deriva del gradual, un corto pasaje de los salmos que se canta detrás de la Epístola; y antifonarios, que contienen las partes corales del Oficio Divino, y cuyo nombre deriva de las antífonas que contienen.

La Pentecostés en una inicial «D» (AB/1922, Fig. 1) está recortada hasta el borde y mide $210 \times 200 \mathrm{~mm}$. Realizada sobre pergamino grueso, lleva al verso un tetragrama en líneas rojas con notación musical cuadrada y texto latino. Corresponde a la antífona de laudes para Pentecostés de un antifonario («Dum complerentur dies pentecostes erant omnes pariter in eodem locon).

Como es frecuente en la pintura gótica italiana la escena se desarrolla delante de una habitación en perspectiva. La Virgen se halla rodeada de los doce apóstoles, entre los que puede reconocerse a San Pedro a la izquierda y a San Pablo a la derecha. Las lenguas de fuego del texto bíblico quedan prolongadas por unas líneas blancas que unen las cabezas de los personajes con un semicírculo en la parte superior ${ }^{3}$.

La combinación de un estilo decididamente bizantino en las figuras con el texto en latín del verso nos lleva a situarla dentro de la escuela paduano-veneciana a comienzos del siglo xIv. La miniatura véneta en la segunda mitad del siglo XIII recoge una fuerte impronta bizantina, que se transforma gradualmente hacia un estilo menos hierático y más naturalista gracias a la influencia del nuevo arte gótico desarrollado en Francia. La obra más característica de este momento es el Epistolario escrito por Giovanni de Gaibana en 1259, que se conserva en la Biblioteca Capitular de la Catedral de Padua ${ }^{4}$, en torno al cual se han agrupado otras dos obras, un Salterio (Cambridge, Fitzwilliam Museum, Ms. 36-1950) y un Misal benedictino procedente de la abadía de Admont en Estiria (Lisboa, Museo Gulbenkian, Ms. LA 222). La inicial AB/1922 deriva de este ambiente, especialmente en la rígida composición simétrica, los tipos faciales y el colorido a base de rojos y azules. Sin embargo debe fecharse con alguna posterioridad ya que se aprecian las influencias boloñesas que caracterizarán a la miniatura veneciana a partir de los años finales del siglo. Estas influencias se concretan en el modelado más simple de los ropajes, en la aparición de una caja espacial en perspectiva y en la decoración del fondo azul de la parte superior a base de roleos blancos. Puede verse un estilo similar en otros códices venecianos de comienzos del Trecento como un Misal de la Biblioteca Marciana de Venecia (Ms.Lat. III.111) 5.

La Conversión de San Pablo en una inicial «S» (AB/1925, Fig. 2) no fue recortada al borde y conserva algún resto de las orlas que rodeaban la página. El fragmento completo mide $316 \times 246 \mathrm{~mm}$. y la inicial $211 \times 190 \mathrm{~mm}$. Lleva al verso un tetragrama con notación musical y texto latino. Procede de un gradual, concretamente del introito de la Misa para la fiesta de la conversión de San Pablo ("Scio cui credidi et certus sum»).

\footnotetext{
3 Esta iconografía aparece en otros códices de influencia bizantina, tanto italianos, como el Coral 11 de la Biblioteca Nazionale de Palermo, f. 54 (Daneu Lattanzi, Angela, Lineamenti di storia della miniatura in Sicilia, Florencia, 1968, fig. 62), como franceses, como el Salterio de la reina Ingeborg (Chantilly, Musée Condé, Ms. 1965, f. 32 v.). También puede señalarse su presencia en el arte románico (capitel de la iglesia de La Daurade en el Musée des Augustins de Toulouse). Véase Réau, Louis, Iconographie de l'Art Chrétien, París, 1955, t. II, v. 2, p. 595.

4 Véase Valagussa, Giovanni, "Alcune novità per il miniatore di Giovanni di Gaibana", en Paragone, 1991, n. ${ }^{\circ} 499$, pp. 3-22, con todas las referencias anteriores.

5 Pirani, E., La miniatura gotica, Milán, 1966, pp. 62-66 y Biblioteca Marciana Venezia, Firenze, 1988, pp. 98-99.
} 
$\mathrm{Al}$ verso puede leerse una anotación moderna: «Nicolo da Bologna, 1332». La atribución parece correcta ya que tanto la forma de la inicial como el resto de orla conservado y, sobre todo, el estilo de las figuras remiten a la figura más importante de la miniatura boloñesa de la segunda mitad del siglo xIv: Niccolò di Giacomo di Nascimbene, llamado Niccolò da Bologna ${ }^{6}$. Aunque se ignora la fecha de su nacimiento, sabemos que trabaja desde 1349 (Libro de Horas, Kremsmünster, Stiftsgal., Clm. 4) hasta su muerte en 1403. Por ello ignoramos a qué puede referirse la fecha 1332, que en ningún caso puede servir para datar la miniatura. Entre su amplia producción, en buena parte firmada y fechada, conservamos numerosos fragmentos recortados que formaron parte de libros de coro.

La forma de la letra «S», que comienza y termina en sendos rostros barbados, fue frecuente en la obra de Niccolò da Bologna, como puede verse en una inicial con San Pedro, (Londres, Colección Wallace, M. 337) ${ }^{7}$, un De Bello Pharsalico de Lucano (Milán, Biblioteca Trivulziana, codice 691, fol. 53 v) ${ }^{8}$ y un gradual (Padua, Biblioteca Antoniana, Libro XII. Bach. YY, 23, fol. 45) ${ }^{9}$. También son muy habituales los roleos blancos que llenan el fondo de la escena y los restos de orla a base de hojas rojas, azules y verdes con pequeños círculos dorados. Sin embargo la mayor ambición compositiva de la escena, el movimiento de las figuras y algunos detalles, como la cabeza de Cristo asomando en la parte superior acercan nuestra inicial a un recorte que estuvo en la Colección Lázaro Galdiano de Madrid ${ }^{10}$ y a una página de coral que estuvo en la Colección Olshki ${ }^{11}$. El estado fragmentario de los tres, unido a la presencia en colecciones españolas de dos de ellos, permite pensar que pertenecieron al mismo libro de coro o, al menos, a una misma serie.

Su estilo nos lleva a fechar estas iniciales en el tercer cuarto del siglo xIv, un margen amplio pero que permite encuadrarlas dentro de la primera parte de la carrera de su autor. En esta inicial Niccolò resuelve la escena sin evitar los condicionantes que impone la forma de la letra, de manera que la «S» separa claramente la figura de San Pablo caído en el suelo y con su atributo, la espada, bien visible, del grupo de sus acompañantes que le contemplan asombrados y de la cabeza de Cristo que en lo alto recrimina al apóstol de los gentiles su persecución. La brillante paleta a base de tonos fríos, azules y rojos, es también muy característica de su autor.

Cristo defendiendo a la mujer adúltera de la lapidación en una inicial «M» $(\mathrm{AB} / 1923$, Fig. 3) está recortada hasta el borde y mide $200 \times 198 \mathrm{~mm}$. El verso lleva también notación musical y texto latino. Los tipos humanos y, sobre todo, la decoración del fondo de la escena a base de roleos vegetales con fuerte relieve y delicadamente dibujados sitúa la miniatura en la escuela lombarda y en los años centrales del siglo xv ${ }^{12}$.

6 Sobre este artista véase D’Ancona, Paolo "Nicolò da Bologna miniaturista del secolo XIV", en Arte Lombarda, XIV, 1969, pp. 1-22; Aeschlimann, Erardo, "Aggiunte a Nicolò da Bologna", ibidem, pp. 23-35; Cassee, Elly, "Illustratori bolognesi del Trecento", en La miniatura italiana in età romanica e gotica: atti del I Congresso di Storia della Miniatura Italiana, Florencia, 1979, pp. 395-418 y Gibbs, R., "Recent developments in the study of bolognese and trecento illustration", en The Burlington Magazine, v. 125, 1984, p. 638 y ss.

7 Alexander, J. J. G., Wallace Collection..., n. 13.

8 Bologna, Giulia, Miniature italiane della Biblioteca Trivulziana, Milán, 1974, p. 56.

9 Abate, Giuseppe y Luisetto, Giovanni, Codici e manoscritti della Biblioteca Antoniana, Vicenza, 1975 , fig. 88.

10 La Colección Lázaro, Madrid, 1926, p. 132. No hemos podido localizar este fragmento entre los fondos de la biblioteca de la Fundación Lázaro Galdiano, donde sí se conserva otro fragmento del mismo autor, una inicial "A" con San Antonio (Inv. 15288, n. ${ }^{\circ}$ 27) (Ibidem, p. 374), que parece de fecha posterior.

11 D'Ancona, Paolo, "Di alcune opere inedite di Nicolò di Giacomo da Bologna» en La Bibliofilia, XIV, 1912 , pp. 281-84, fig. 4 .

12 Encontramos fondos decorados de manera similar en otras obras lombardas del mismo momento, como el Liber iudiciorum de Raphael de Vicomercato, Milán, Biblioteca Trivulziana, cod. 1329 (Santoro, Caterina, I codici miniati della Biblioteca Trivulziana, Milán, 1958, n. ${ }^{\circ}$ 24), una inicial conservada en la State Library of Victoria (Manion, M. y Vines, Vera F., Medieval and renaissance illuminated manuscripts in Australian collections, Melbourne, 1984, n. ${ }^{\circ}$ 19, p. 74) o una inicial atribuida a un seguidor de Stefano da Zevio conservada en el Metropolitan Museum de Nueva York (Hibbard, Howard, The Metropolitan Museum of Art, Nueva York, 1980, fig. 392). 

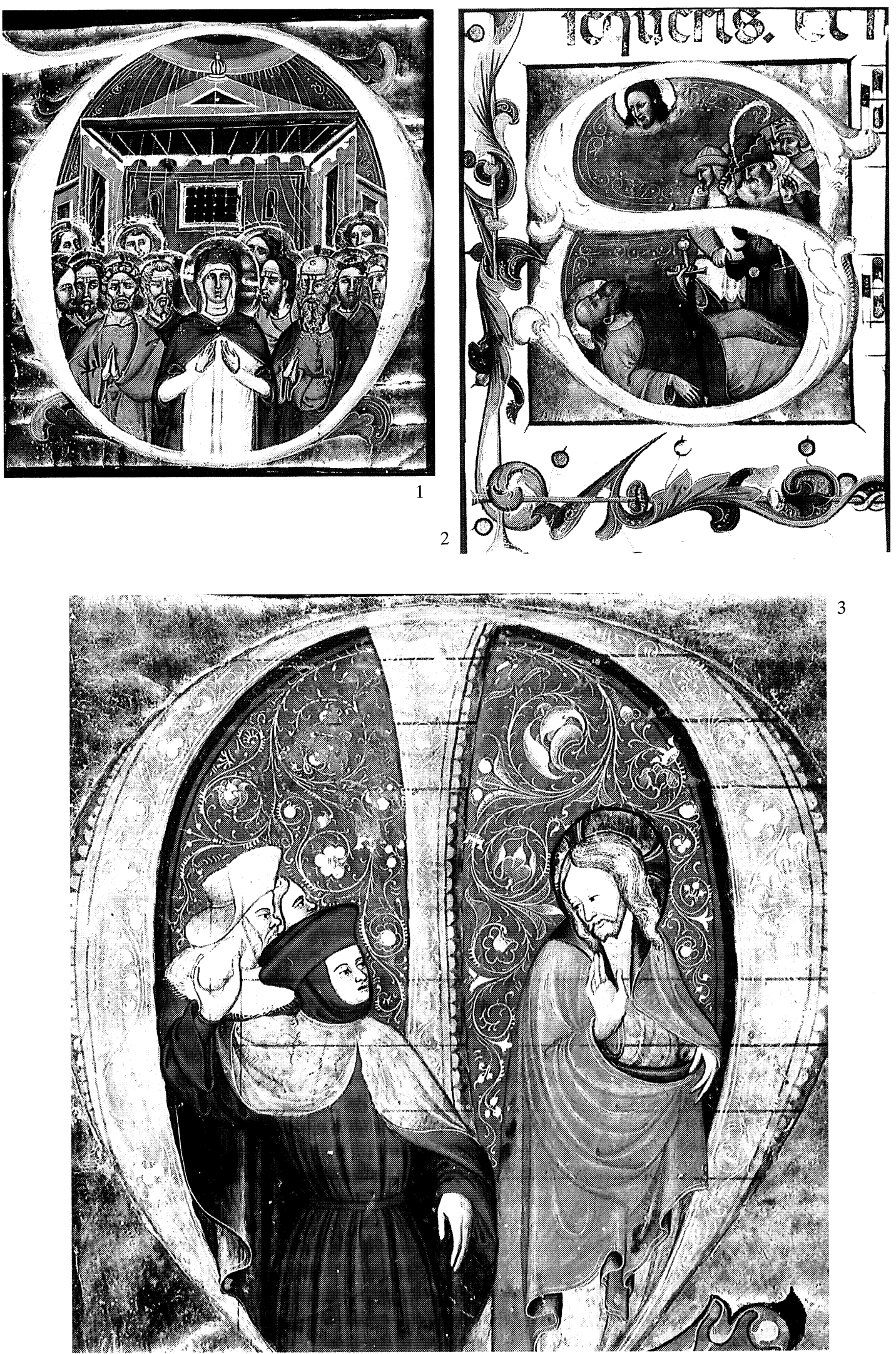

Fig. 1. Escuela paduano-veneciana. Pentecostés. Madrid, Biblioteca Nacional.

Fig. 2. Niccoló da Bologna, Conversión de San Pablo. Madrid, Biblioteca Nacional.

Fig. 3. Escuela lombarda. Cristo defendiendo a la mujer adúltera. Madrid, Biblioteca Nacional. 
La miniatura lombarda se configura a lo largo del siglo xIv bajo la influencia de la escuela boloñesa. A finales de siglo el mecenazgo de los Visconti abre el arte lombardo a los influjos transalpinos y supone para la iluminación de libros un momento de gran esplendor. Entre los iluminadores destacan a finales de siglo Giovannino de Grassi y Michelino da Besozzo. De la obra de ambos, dentro del gótico internacional, derivan los maestros anónimos de la primera mitad del siglo xv como el Maestro de las Vitae Imperatorum, el Maestro del Breviario Franciscano y el Maestro del Antifonario de Budapest.

Podemos encuadrar nuestra miniatura dentro del círculo del Maestro de las Vitae Imperatorum, nombre acuñado por Pietro Toesca a partir del códice de Suetonio de la Bibliothèque Nationale de París (ms. it. 131), realizado en 1431 para Filippo Maria Visconti ${ }^{13}$. Trabajó en una numerosa serie de manuscritos entre 1408 y 1459, de los que en España sólo se ha identificado un códice con las Sátiras de Filelfo, fechado en 1453 (Valencia, Biblioteca General de la Universidad, Ms. 398). Su estilo, que ha sido confundido en alguna ocasión con el de otros maestros, tuvo gran influencia en la miniatura lombarda del siglo xv. Se caracteriza por los tipos humanos de rostros descarnados con los ojos muy marcados, colorido fuerte y contrastado, y fondos lisos con decoraciones vegetales. Todos estos rasgos aparecen en esta miniatura, que, sin embargo, no creemos que sea obra del propio maestro sino de algún seguidor, que también realizó un fragmento de un antifonario conservado en la Free Library de Filadelfia (M.27.21). Representa la Entrada de Cristo en Jerusalén, mide $190 \times 140 \mathrm{~mm}$ y se desconoce su origen ${ }^{14}$. Ambos fragmentos comparten, además de la decoración del fondo de la escena ya señalada, unos personajes con rasgos similares y detalles peculiares como los dedos de remate puntiagudo. Asimismo la técnica empleada es parecida ya que en los dos casos las líneas del tetragrama subyacente afloran en la miniatura. Por su estilo pueden fecharse en los años centrales del siglo xv, más concretamente en el período 1445-1450, momento en el que, según Anna Melograni 15, los códices de este taller muestran una técnica más acuarelada y menos cubriente.

F. JAVIER DocAmpo Biblioteca Nacional

\section{MÚSICA Y RETÓRICA EN LA ALEGORÍA DEL OÍDO DE JAN BRUEGHEL «DE VELOURS» Y RUBENS}

La Alegoría del oído es una tabla sin firma, pintada hacia 1617 por Jan Brueghel (1568$1625)$ en colaboración con Pedro Pablo Rubens (1577-1640). Forma parte de una colección sobre los cinco sentidos realizada para los Archiduques de Austria que se custodia actualmente en

13 Toesca, P., La pittura e la miniatura nella Lombardia dai piú antichi monumenti alla metà del Quattrocento, Milán, 1912 , p. 529. Sobre este maestro véase además Stefani, Letizia, "Per una storia della miniatura lombarda da Giovannino de Grassi alla scuola cremonese della II metà del quattrocento: appunti bibliografici", en La miniatura italiana tra Gotico e Rinascimento: atti del II Congresso di Storia della Miniatura Italiana, Florencia, 1985, pp. 838-841 y 875-881; Stones, Alison, "An italian miniature in the Gambier-Parry collection" en The Burlington Magazine, CXI, 1969, pp. 7-12; Toesca, Ilaria, "In margine al Maestro delle Vitae Imperatorum» en Paragone, XX, n. ${ }^{\circ} 237,1969$, pp. 73-77 y Melograni, Anna, "Appunti di miniatura lombarda: ricerche sul Maestro delle Vitae Imperatorum», en Storia dell'Arte, n. ${ }^{\circ}$ 170, 1990, pp. $274-314$.

14 Daneu Lattanzi, Angela, "La miniatura nella Italia Meridionale» en La miniatura italiana tra gotico e rinascimento: atti del II Congresso di Storia della Miniatura italiana, Florencia, 1985, pp. 775-776, fig. 16. Agradezco a Karen Lightner, de la Free Library, las informaciones proporcionadas sobre este fragmento.

15 Melograni, Anna, Op. cit., pp. 296-98. 